\title{
EFECTIVIDAD DE LOS CONTROLES PRENATALES EN GESTANTES ATENDIDOS EN EL HOSPITAL REGIONAL DE ICA, PERÚ.
}

\author{
EFFECTIVENESS OF THE PRENATAL CONTROLS IN PREGNANT WOMEN \\ ATTENDED AT THE REGIONAL HOSPITAL OF ICA, PERÚ.
}

\author{
1. Universidad Particular San Juan Bautista. Filial-Ica. \\ a. Cirujano General. \\ b. Médico Cirujano \\ c. Médico Internista.
}

Leveau-Bartra Harry ${ }^{1, a}$, Vásquez-Morales Vladimiro Pabel ${ }^{1, b}$, Vásquez-Morales Mirko ${ }^{1, c}$

DOI: https://doi.org/10.35563/rmp.v8i3.191

Correspondencia:

Leveau-Bartra Harry.

Número de celular: 956608888

Correo Electrónico:

kahaisail4@hotmail.com

\section{Contribuciones De Autoría:}

$\mathrm{LBH}, \mathrm{VMV}$, VMM, participaron en el diseño del estudio, el análisis de los datos, revisaron críticamente el artículo y aprobaron la versión final.

Conflicto De Intereses: No declarados.

Financiamiento: Autofinanciado.

\section{Como Citar}

Leveau Bartra Harry, Vásquez Morales Vladimiro Pabel, Vásquez MoralesMirko. Efectividad de los Controles Prenatales en gestantes atendidos en el Hospital Regional de Ica, Perú. Rev méd panacea. 2019; 8(3): 90-95. DOI: https://doi.org/10.35563/rmp.v8i3.191

Recibido: 17 - 08 - 2019

Aceptado: 20 - $09-2019$

Publicado: 22 - 09 - 2019

\section{RESUMEN}

Objetivo: Evaluar la efectividad de los Controles Prenatales (CPN) en gestantes atendidos en el Hospital Regional de Ica febrero a mayo 2019. Materiales y métodos: Observacional, trasversal, retrospectiva de casos y controles, Nivel relacional, diseño de casos y controles. Muestra 126 gestantes atendidos en el Hospital Regional de Ica entre febrero y mayo del 2019, seleccionada por muestreo a criterio de la investigación. Resultados: Los controles prenatales en número de 6 veces a más no son efectivos en disminuir el tiempo del periodo expulsivo en gestantes atendidos en el referido hospital de febrero a mayo 2019. Los controles prenatales en número de 6 veces a más no son efectivos en disminuir la probabilidad de retención de restos placentarios en gestantes atendidos en dicho hospital de Ica de febrero a mayo 2019. Los Controles Prenatales en número de 6 veces a más no son efectivos en disminuir la probabilidad de presentar desgarros vaginales en gestantes atendidos en el Hospital Regional de Ica febrero a mayo 2019. Los Controles Prenatales en número de 6 veces a más son efectivos en mejorar el Apgar del Recién Nacido en gestantes atendidos en el Hospital Regional de Ica febrero a mayo 2019. Conclusiones: Los controles prenatales de 6 a más (Gestante controlada) no disminuye el tiempo de expulsivo, tampoco disminuye la probabilidad de presentar retención de restos placentarios ni desgarros vaginales, y si disminuye la morbimortalidad del Recién Nacido al influir en mejorar el Apgar del Recién Nacido.

Palabras clave: Efectividad; atención prenatales; Perú (fuente: DeCS BIREME).

\begin{abstract}
Objective: To evaluate the effectiveness of the Prenatal Controls in pregnant women attended in the Regional Hospital of Ica February to May 2019. Materials and methods: Observational, cross-sectional, retrospective of cases and controls, relational level, case and control design. Sample 126 pregnant women attended at the Regional Hospital of Ica between February and May of 2019, selected by sampling at the discretion of the research. Results: Prenatal controls in number of 6 times more are not effective in reducing the time of the expulsive period in pregnant women attended at the Regional Hospital of Ica from February to May 2019. Prenatal check-ups in number of 6 times are not effective in reducing the probability of retention of placental remains in pregnant women attended at the Regional Hospital of Ica from February to May 2019. Prenatal Controls in number of 6 times more are not effective in reducing the probability of presenting vaginal tears in pregnant women treated at the Regional Hospital of Ica from February to May 2019. The Prenatal Controls in number of 6 times more are effective in improving the Apgar of the Newly Born in pregnant women attended at the Regional Hospital of Ica February to May 2019. Conclusions: Prenatal check-ups from 6 to more (Controlled Gestant) does not decrease the expulsion time, nor does it diminish the probability of presenting retention of placental remains or vaginal tears, and if the Newborn morbidity and mortality decreases as it influences in improving the Apgar of the Newborn.
\end{abstract}

Keywords: Effectiveness; prenatal care; Peru (source: MeSH NLM). 


\section{INTRODUCCIÓN}

Los Controles Prenatales son procedimientos que se realizan para prevenir patologías durante la gestación y preparar a las gestantes en el proceso de gestación y parto la que debería manifestarse en menores complicaciones durante la gestación y durante el parto y puerperio, así mismo detecta patologías precozmente la que posibilita un tratamiento oportuno en benéficio de la madre y del recién nacido (1). Las recomendaciones de la Organización Mundial de la Salud 2016 sobre atención prenatal para una experiencia de embarazo positiva priorizan la atención de la salud centrada en la persona, el bienestar de las mujeres y las familias, y los resultados maternos y perinatales positivos (2). Para ello es necesario que dichos controles prenatales renfocados cumplan con las normas técnicas impartidas por el Ministerio de Salud (3). Debe considerarse efectivo un control prenatal cuando éste es precoz, periódico e integral, entendiéndose como precoz cuando se realiza desde la sospecha de embarazo; periódico cuando es mensual hasta las 32 semanas de gestación y cada 15 días hasta los 37 semanas y luego de ello 1 vez por semana hasta el final, e integral cuando las atenciones son realizadas por diferentes profesionales a fin de tener variados y mejores puntos de vista (4). Así mismo la $1^{\circ}$ consulta de atención prenatal no será menor a 40 minutos y las siguientes no menos de 25 minutos y debe ser realizada por profesional Gineco-Obstetra u Obstetra (5).

Por ello la OMS recomienda que los controles prenatales incluyan intervenciones en el sistema de salud para mejorar el uso y la calidad de la atención prenatal, y la experiencia positiva del embarazo de mujeres y adolescentes, con una recomendación de un mínimo de ocho contactos de atención prenatal durante el embarazo para mejorar los resultados perinatales y la experiencia de las mujeres en la atención, estas recomendaciones están diseñadas para ser adaptables y flexibles, de modo que los países con diferentes entornos, cargas de morbimortalidad, situaciones sociales y económicas, y estructuras de sistemas de salud puedan adoptar e implementar las recomendaciones basándose en el contexto de su país y en las necesidades de sus habitantes (2). Sin embargo, en Huaral y Chancay, Rivera Félix L. (6) determina que el olvido de las citas y la presencia de un seguro de salud previo al embarazo están relacionadas con la no realización de CPN.

El objetivo del modelo de atención prenatal de la OMS de 2016 es ofrecer a las embarazadas atención respetuosa, individualizada y centrada en la persona en cada contacto, y asegurar que cada uno se apoye en prácticas clínicas eficaces e integradas (intervenciones y análisis), aporte información pertinente y oportuna, y ofrezca apoyo psicosocial y emocional por parte de profesionales con buenas habilidades clínicas e interpersonales en un sistema de salud que funcione correctamente.

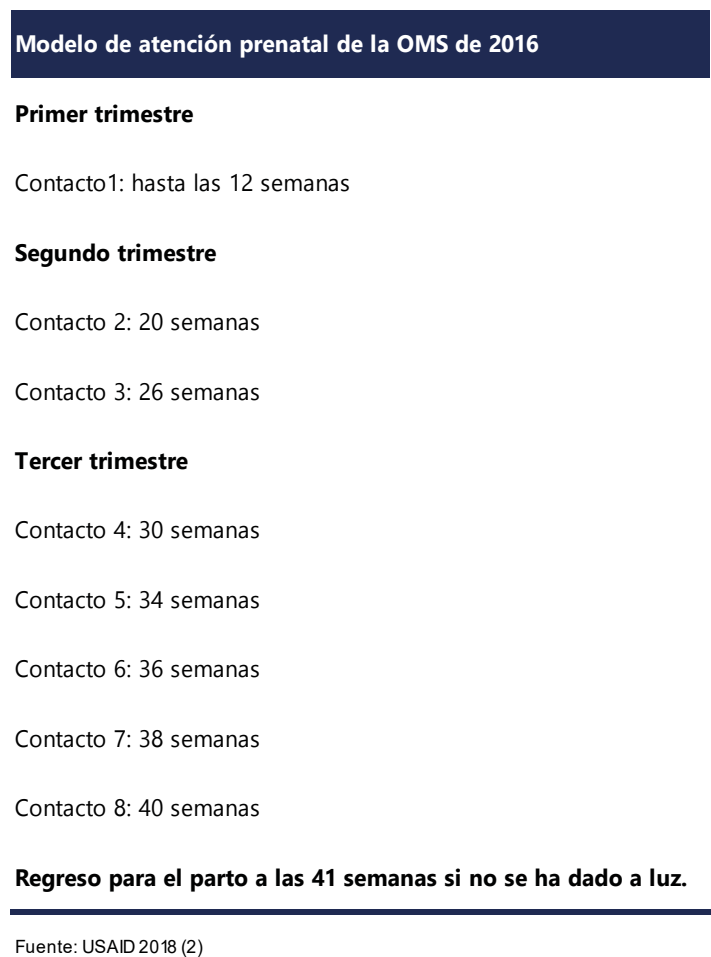

En el Hospital Regional de Ica se atiende en promedio 110 partos vaginales por mes por lo que es interés conocer la frecuencia de gestantes que tienen controles prenatales, así como su efectividad en el tiempo de expulsivo, en la presencia de desgarros, en la retención de restos placentarios y en el Apgar del Recién Nacido. Para ello se evaluó estos parámetros en las gestantes que tuvieron parto durante los meses de febrero a mayo del 2019

\section{MATERIALES Y MÉTODOS \\ Tipo de investigación}

Observacional, trasversal, retrospectiva analítica

\section{Nivel y Diseño de estudio}

Nivel relacional cuyo objetivo es comparar. Diseño Representado como:

\begin{tabular}{cccc} 
Número de CPN & Anormal & Normal & Total \\
\hline 5 a menos CPN & $a$ & $B$ & $a+b$ \\
6 a más CPN & $c$ & $D$ & $c+d$ \\
Total & $a+c$ & $b+d$ & $a+b+c+d$ \\
\hline
\end{tabular}

\section{Población}

Está conformada por gestantes que tuvieron parto entre febrero y mayo del 2019 en el Hospital Regional de Ica.

\section{Muestra}

Todas las gestantes que tienen datos completos y 
legibles en la historia clínica y fueron atendidos en el Hospital Regional de Ica entre febrero y mayo del 2019.

MUESTREO. Según criterio de la investigación: Gestante que cuente con datos completos y legibles en la historia clínica.

Unidad de análisis. Gestante con historia clínica completa y legible que fue atendida su parto vía vaginal en el Hospital Regional de Ica.

\section{Hipótesis}

- H1: Los controles prenatales en número de 6 veces a más son efectivos en disminuir el tiempo del periodo expulsivo en gestantes.

- H1: Los controles prenatales en número de 6 veces a más son efectivos en disminuir la probabilidad de retención de restos placentarios

- H1: Los Controles Prenatales en número de 6 veces a más son efectivos en disminuir la probabilidad de presentar desgarros vaginales

- H1: Los Controles Prenatales en número de 6 veces a más son efectivos en mejorar el Apgar del Recién Nacido

\section{Variables}

VARIABLE INDEPENDIENTE

- Controles prenatales

VARIABLE DEPENDIENTE

- Tiempo del expulsivo

- Retención de restos placentarios

- Desgarros vaginales

- Apgar del Recién Nacido

\section{RESULTADOS}

Tabla 1: Clasificación de las gestantes por número de controles prenatales.

\begin{tabular}{cccc} 
CPN & Frecuencia & Porcentaje & $\begin{array}{c}\text { Porcentaje } \\
\text { acumulado }\end{array}$ \\
\hline 0 & 7 & $5,60 \%$ & $5,60 \%$ \\
1 & 4 & $3,20 \%$ & $8,70 \%$ \\
\hline 2 & 3 & $2,40 \%$ & $11,10 \%$ \\
3 & 7 & $5,60 \%$ & $16,70 \%$ \\
4 & 2 & 1,6 & $18,30 \%$ \\
5 & 6 & $4,80 \%$ & $23,00 \%$ \\
\hline 6 & 22 & $17,50 \%$ & $40,50 \%$ \\
7 & 13 & $10,30 \%$ & $50,80 \%$ \\
\hline 8 & 21 & $16,70 \%$ & $67,50 \%$ \\
\hline 9 & 18 & $14,30 \%$ & $81,70 \%$ \\
10 & 18 & $14,30 \%$ & $96,00 \%$ \\
11 & 4 & $3,20 \%$ & $99,20 \%$ \\
\hline 12 & 1 & $0,80 \%$ & $100,00 \%$ \\
\hline & 126 & $100,00 \%$ & \\
\hline
\end{tabular}

Tabla 2: Diferencia de medias en el tiempo del expulsivo

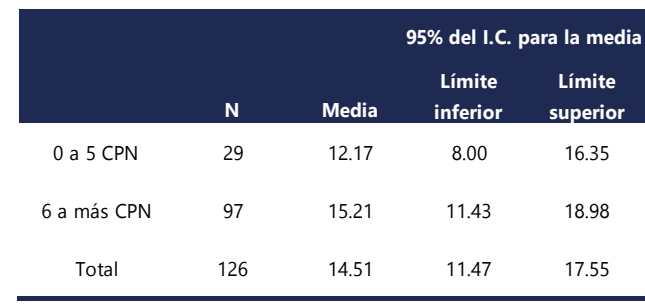

Fuente: Hospital regional de Ica febrero-mayo 2019.

Tabla 3: $t$ de Student para diferencia de medias en el tiempo del expulsivo

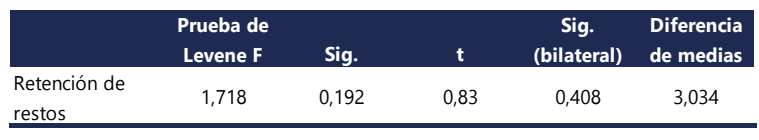

Fuente: Hospital regional de Ica febrero-mayo 2019

Tabla 4: Retención de restos placentarios en relación a los controles prenatales

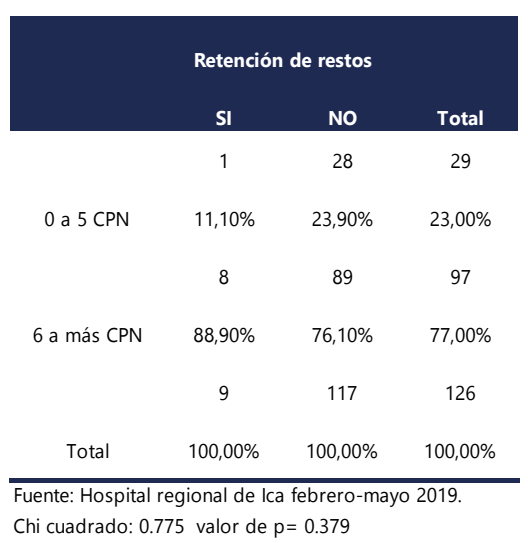

Tabla 5: Desgarro vaginales en relación a los controles prenatales

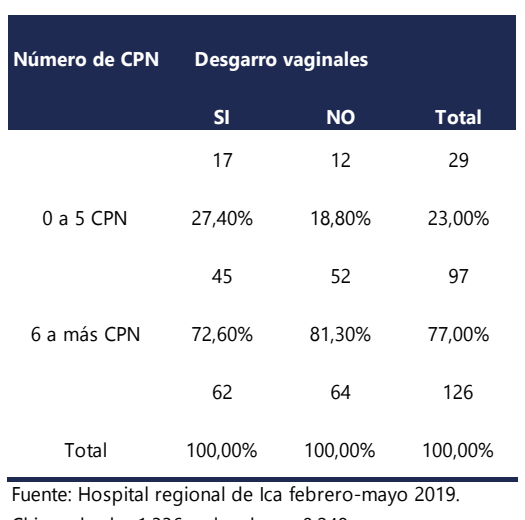

Chi cuadrado: 1.336 valor de $p=0.248$ 
Tabla 6: Apgar del Recién Nacido en relación a los controles prenatales.

\begin{tabular}{|c|c|c|c|}
\hline & \multicolumn{2}{|c|}{ APGAR } & \multirow[b]{2}{*}{ Total } \\
\hline & $\begin{array}{c}\text { Apgar de } 8 \\
\text { a menos }\end{array}$ & $\begin{array}{c}\text { Apgar } 9 \text { a } \\
\text { más }\end{array}$ & \\
\hline \multirow{3}{*}{0 a 5 CPN } & 4 & 25 & 29 \\
\hline & $57,10 \%$ & $21,00 \%$ & $23,00 \%$ \\
\hline & 3 & 94 & 97 \\
\hline \multirow{2}{*}{6 a más $C P N$} & $42,90 \%$ & $79,00 \%$ & $77,00 \%$ \\
\hline & 7 & 119 & 126 \\
\hline Total & $100,00 \%$ & $100,00 \%$ & $100,00 \%$ \\
\hline
\end{tabular}

\section{PRUEBA DE HIPÓTESIS}

\section{HIPÓTESIS ESPECÍFICA 1}

1.-Formulación de las hipótesis

Ha: Los Controles Prenatales en número de 6 veces a más son efectivos en disminuir el tiempo del periodo expulsivo.

Ho: Los Controles Prenatales en número de 6 veces a más No son efectivos en disminuir el tiempo del periodo expulsivo.

2.- Nivel de significación: $95 \%$

3.-Estadística de prueba. - t Sudent sin asumir varianzas iguales según la prueba de Levene

4.- Valor de t Calculado $=1,1 \quad$ Valor de $p=$ 0.28

5.- Interpretación: Como el valor de $\mathrm{p}$ es mayor de 0.05 no se puede rechazar Ho: Los Controles Prenatales en número de 6 veces a más No son efectivos en disminuir el tiempo del periodo expulsivo.

6.-Conclusión. - Los controles prenatales no influyen en disminuir el tiempo del periodo expulsivo en gestantes atendidos en el Hospital Regional de Ica febrero a mayo 2019

\section{HIPÓTESIS ESPECÍFICA 2}

1.-Formulación de las hipótesis

Ha: Los controles prenatales en número de 6 veces a más son efectivos en disminuir la probabilidad de retención de restos placentarios

Ho: Los controles prenatales en número de 6 veces a más No son efectivos en disminuir la probabilidad de retención de restos placentarios

2.- Nivel de significación: $95 \%$

3.-Estadística de prueba. - Chi cuadrado

4.-Valor de t Calculado $=0.775 \quad$ Valor de $p=$

\subsection{9}

5.- Interpretación: Como el valor de p es mayor de 0.05 no se puede rechazar Ho: Los controles prenatales en número de 6 veces a más No son efectivos en disminuir la probabilidad de retención de restos placentarios
6.-Conclusión. - Los controles prenatales no influyen en disminuir la probabilidad de retención de restos placentarios en gestantes atendidos en el Hospital Regional de Ica febrero a mayo 2019.

\section{HIPÓTESIS ESPECÍFICA 3}

\section{1.-Formulación de las hipótesis}

Ha: Los Controles Prenatales en número de 6 veces a más son efectivos en disminuir la probabilidad de presentar desgarros vaginales

Ho: Los Controles Prenatales en número de 6 veces a más no son efectivos en disminuir la probabilidad de presentar desgarros vaginales

2.- Nivel de significación: $95 \%$

3.-Estadística de prueba. - Chi cuadrado

4. - Valor de $\mathrm{t}$ Calculado $=1.336$

Valor de $\mathrm{p}=0.248$

5.- Interpretación: Como el valor de p es mayor de 0.05 no se puede rechazar Ho: Los Controles Prenatales en número de 6 veces a más No son efectivos en disminuir la probabilidad de presentar desgarros vaginales.

6.-Conclusión. - Los controles prenatales no influyen en disminuir la probabilidad de presentar desgarros vaginales en gestantes atendidos en el Hospital Regional de Ica febrero a mayo 2019

\section{HIPÓTESIS ESPECÍFICA 4}

\section{1.-Formulación de las hipótesis}

Ha: Los Controles Prenatales en número de 6 veces a más son efectivos en mejorar el Apgar del Recién Nacido Ho: Los Controles Prenatales en número de 6 veces a más no son efectivos en mejorar el Apgar del Recién Nacido

2.- Nivel de significación: $95 \%$

3.-Estadística de prueba. - Chi cuadrado

4.- Valor de t Calculado $=4,872$

Valor de $\mathrm{p}=0.027$

5.- Interpretación: Como el valor de p es menor de 0.05 se rechaza Ho y se acepta Ha: Los Controles Prenatales en número de 6 veces a más son efectivos en mejorar el Apgar del Recién Nacido

6.-Conclusión. - Los controles prenatales influyen en tener un Recién Nacido con Apgar de 9 a más a los 5 minutos, en gestantes atendidos en el Hospital Regional de Ica entre febrero a mayo 2019

\section{DISCUSIÓN}

En la tabla 1 se determina que la proporción de gestantes que cumplen con tener 6 controles prenatales a más es de $77 \%$ siendo inferior a lo estipulado por el Ministerios de Salud (7) que indica que debe ser 100\% y en número de 6 controles a más para considerarlo gestante controlada. Es de mencionar que no fue posible definir la calidad de los mismos. Considerando que los controles prenatales están orientadas además a disminuir las tasas de cesáreas en nuestra realidad se observa que ello no se produce pues continuamos teniendo altas tasas de cesáreas en el hospital regional 
de Ica. Estudios recientes realizados en la Región Metropolitana de Chile y a nivel nacional, revelaron una alta tasa de intervenciones obstétricas, incluida una alta tasa de cesáreas en Chile (8).

En la tabla 2 y 3 se concluye que los controles prenatales no influyen en disminuir el tiempo del periodo expulsivo pues la probabilidad de error al afirmar lo antes mencionado es de $40.8 \%$, corroborado con la intersección que presentan los intervalos de confianza al $95 \%$ observado en la tabla 2. En un estudio realizado por Delgado Lajones, V (9) en Ecuador demuestra que el número inadecuado de controles prenatales se asocia a expulsivo prolongado (16.7\%).

La investigación determinó que la retención de restos placentarios no está influenciada por el número de controles prenatales (gestantes controladas: 6 a más controles prenatales) como se observa en la tabla 4, pues la retención de restos obedece sobre todo a otros factores. Aunque en un estudio desarrollado por Flores Ayala, B (10). y Olivera K. (11) en el Hospital de Cajatambo, concluye que los factores de riesgo para desarrollar complicaciones obstétricas son mujeres mayores de 34 años, multíparas y controles prenatales inadecuados.

En relación a los desgarros vaginales y los controles prenatales, como se observa en la tabla 5 se determinó que el número de controles prenatales no protege contra los desgarros pues estas patologías están en relación sobre todo a macrosomía, primigestas, partos precipitados (12). Sin embargo, Torres Montoya, O. (13) concluye en su estudio que son indicadores epidemiológicos para desgarro perineal, un número no adecuado de controles prenatales y la edad materna mayor a 35 años, la que se debería a la calidad de los controles prenatales que en este estudio no fueron evaluados, pues para que el control prenatal sea efectivo debe ser adecuado en número y en calidad.

En la tabla 6 se determinó que el Apgar del Recién Nacido se ve mejorado cuando una gestante cumple con 6 controles a más pues en el grupo de gestantes controladas (6 controles a más) se observa mayor proporción de recién nacidos con Apgar 9 a 10 luego de los 5 minutos de nacido, resultado que finalmente indicaría la efectividad de los controles prenatales en la morbimortalidad de los recién nacidos que se debería a que estos procedimientos tienen por finalidad prevenir patologías en la gestación (12), tratar oportunamente patologías de la gestante y mejorar la salud física y mental de la gestante en beneficio de un recién nacido que tendrá un mejor Apgar al nacer. Así lo demuestra en su estudio Llallahui Arango, S. (14) en Cañete cuyos resultados demuestran que existe relación entre la atención prenatal reenfocada y los resultados del Apgar en la evaluación inmediata del recién nacido. resultado corroborado por el estudio realizado por Avelino Villon, N.(15) comprueba que el CPN inadecuado es un riesgo para presentar alguna morbilidad neonatal, los neonatos que presentan un inicio tardío del control prenatal tienen mayor probabilidad de presentar morbimortalidad neonatal que aquellos neonatos que presentan menos de 6 controles prenatales.

\section{CONCLUSIONES}

Los controles prenatales de 6 a más (gestante controlada) no disminuye el tiempo de expulsivo, tampoco disminuye la probabilidad de presentar retención de restos placentarios ni desgarros vaginales, y si disminuye la morbimortalidad del recién nacido al influir en mejorar el Apgar del Recién Nacido. 


\section{REFERENCIAS BIBLIOGRÁFICAS}

1. Ministerio de Salud. Dirección General de Servicios de Salud. Normativa - 011. Normas y Protocolos para la Atención Prenatal, Parto, Puerperio y Recién Nacido/a de Bajo Riesgo

2. USAID. 2018. Recomendaciones de la OMS sobre atención prenatal para una experiencia positiva del embarazo: resumen. Disponible en: https://www.mcsprogram.org/wp-content/uploads/... /ANC-OverviewBriefer-A4-SP.pd...

3. Aguilar Esenarro, L. Consejería nutricional en el marco de la atención integral de salud de la gestante y puérpera: guía técnica / Lima: Ministerio de Salud, Instituto Nacional de Salud, 2016.

4. NTS No 105-MINSA-DGSP-V.01: "Norma Técnica de Salud para la Atención Integral de Salud Materna" según RM No 827-2013-MINSA

5. Establecen precisiones y modifican la Norma Técnica de Salud para la Atención Integral de Salud Materna. RESOLUCIÓN MINISTERIAL N ${ }^{\circ}$ 159-2014/MINSA

6. Rivera Felix LM, Burgos López NH, Gomez Diaz JZ, Moquillaza Alcántara VH. Factores asociados al acceso a la atención prenatal en los hospitales de Huaral y Chancay, Perú. An Fac med. 2018;79(2):131-37. DOI: http://dx.doi.org/10.15381/anales.v79i2.14939.

7. Establecen precisiones y modifican la Norma Técnica de Salud para la Atención Integral de Salud Materna. RESOLUCIÓN MINISTERIAL $\mathrm{N}^{\circ}$ 159-2014/MINSA

8. Manual de talleres prenatales en la Atención Primaria de Salud: Una herramienta para matronas y matrones. Programa de Salud de la Mujer- Chile Crece Contigo.

9. Delgado Lajones, V. Factores que desencadenan parto distócico en las embarazadas que se asisten al Hospital General Sur Delfina Torres de Concha.
Ecuador. Disponible en: D Lajones, V Solange - 2019 - repositorio.pucese.edu.ec

10. Flores Ayala, B. Factores de riesgo para desarrollar complicaciones obstétricas en puérperas mayores de 34 años en el Hospital Barranca Cajatambo durante el año 2018. Disponible en: BA Flores Ayala - 2019 - repositorio.upsjb.edu.pe

11. Olivera Campos, K. Caracteristicas sociodemográficas y obstétricas de puérperas que presentaron retención placentaria en el centro de salud de el tambo 2017 y 2018. Disponible en: O Campos, K Guadalupe - 2019

12. repositorio.unh.edu.pe 12. MINSA. Plan nacional para la reducción de la mortalidad materna y Perinatal. Situación actual de

13. la salud sexual y reproductiva monitoreo y evaluación de suministros. Disponible en:

$\mathrm{ftp}: / / \mathrm{ftp} . \mathrm{minsa.gob} . \mathrm{pe} / \mathrm{sismed} / \mathrm{ftp}$ carga/...15\%20M ARZO\%202018/.../SSR.pdf

14. 13.- Torres Montoya, O. Indicadores epidemiológicos asociados a desgarros perineales en gestantes atendidas en el servicio de gineco-obstetricia del Hospital Santa Rosa durante el periodo Junio 2017 - Junio 2018. Disponible en.

15. OV Torres Montoya - 2019 - repositorio.urp.edu.pe 14.- Llallahui Arango, S. Atención Prenatal Reenfocada y los resultados en la evaluación inmediata del recién nacido en madres adolescentes de 12-17 años en el Centro de Salud Mala - Cañete durante el año 2014 -2016

15.- Avelino Villon, N. Control prenatal inadecuado como factor de riesgo para la morbimortalidad neonatal en un hospital de referencia de lima octubre - diciembre 2018. 